\title{
The Spreading of an Insoluble Surfactant on a Thin Non-Newtonian Fluid
}

\author{
MATTHEw MCCURDY \\ Centre College, Department of Mathematics \\ e-mail: matthew.mccurdy@centre.edu \\ Supervisor: Dr. Ellen Swanson \\ Centre College, Department of Mathematics \\ e-mail: ellen.swanson@centre.edu
}

\begin{abstract}
In this paper, we explore fluid flow caused by the presence of an insoluble surfactant on a thin, incompressible power-law fluid over a horizontal substrate. The gradient in surface tension caused by the surfactant results in fluid flow away from the region where the surfactant was deposited. Work has been conducted with Newtonian fluids and surfactants; however, the extensive effect surfactants have on non-Newtonian fluids has not been studied as thoroughly.

Using the lubrication approximation, we derive a system of coupled nonlinear partial differential equations (PDE) governing the evolution of the height of the fluid and the spreading of the surfactant. We also numerically simulate our system with a finite difference method and vary the power-law index to explore differences in profiles of shear-thickening and shear-thinning fluids. Next, we find significant agreement between our results and previous studies involving Newtonian fluids with power-law relations. Finally, we determine similarity scalings and solutions around the leading edge of the surfactant, which describe the behavior of the fluid and surfactant towards the region of the fluid where the surfactant ends.
\end{abstract}

\section{Introduction}

Surfactants, compounds which lower surface tension, have been investigated in conjunction with fluids due to the flow they cause. The local changes in surface tension caused by surfactants results in flow, known as Marangoni flow, away from where the surfactant was deposited. This flow has been studied extensively with Newtonian fluids, but not as much headway has been made with non-Newtonian fluids and surfactants. Jensen, Gaver, Grotberg, and Naire studied the spreading of surfactant on thin liquid films as they determined the equations and investigated properties of solutions analytically and numerically in a series of papers $[7,8,9,10,11,12]$. Other researchers have studied the same fluid flow with simulations $[4,15,18]$ and experiments $[2,6]$. While non-Newtonian fluids, characterized by their nonlinear stress-strain relationships or non-zero yield stress, do not lend themselves to the more elegant, concise arguments of their Newtonian counterparts, there are still innumerable practical applications of non-Newtonian fluids. In nature, the slow flow of non-Newtonian fluids is found in the lining of human lungs [20], the flow of molten liquids [3], and the movement of microorganisms [13]. In medicine, understanding more fully how surfactants interact with non-Newtonian fluids has the 


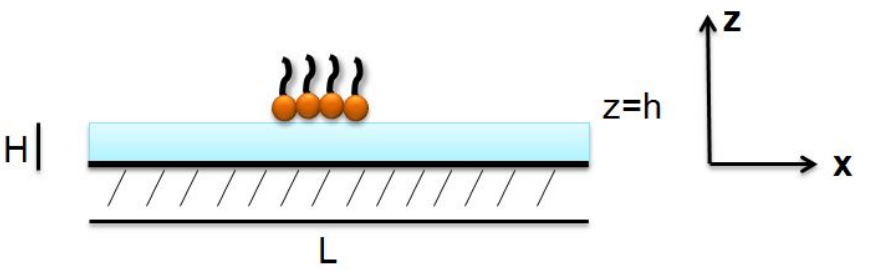

Figure 1: A thin fluid over a solid, horizontal substrate with a drop of an insoluble surfactant. The height scale, $H$, is assumed to be much less than the length scale, $L$.

potential to aid in alleviating symptoms of cystic fibrosis and even to increase effectiveness of treating respitory distress syndrome with surfactant replacement therapy $[22]$.

In this work, we investigate how an insoluble surfactant spreads on a thin, incompressible non-Newtonian fluid with a power-law relation on a horizontal substrate. In Section 2, we apply the lubrication approximation to derive a system of nonlinear partial differential equations (PDE) governing the evolution of the height of the fluid and the spreading of the surfactant. We non-dimensionalize the system and the associated boundary and initial conditions in preparation to numerically simulate the system with a finite difference method in Section 3. We find agreement between our results and previous studies conducted with Newtonian fluids [7, 9, 18, 19]. Additionally, we vary the power-law index to see the effect it has on the height and surfactant profiles and determine similarity scalings in Section 4.

\section{The Model}

Consider a thin, incompressible non-Newtonian fluid on a horizontal substrate with an insoluble surfactant, as shown in Figure 1, where the height of the fluid, $z=h(x, t)$, is initially uniform. The surfactant sits on top of the fluid and the concentration of the surfactant is denoted as $\Gamma=\Gamma(x, t)$. Motivated by the droplet of surfactant and fluid spreading evenly from the center of where the surfactant was deposited, we consider two dimensional spreading: $x$ for length and $z$ for height.

With thin fluids, a general assumption made is that the height scale $H$ is much smaller than the length scale $L$, or $H \ll L$. We assume $\frac{H}{L}=\epsilon \ll 1$. This is referred to as the lubrication approximation, and aids in discerning dominant terms when deriving the governing equations. Another condition we assume is that the fluid has a slow flow. This assumption follows from the lubrication approximation and allows inertial effects on the fluid's flow to be ignored.

In this section, we derive the system of nonlinear PDE governing the evolution of the height of the fluid and spreading of the surfactant.

\subsection{Deriving Equations}

Perazzo and Gratton [17] derived the governing equations for a thin, incompressible power-law fluid flowing down an incline. However, their work was in three dimensions, incorporated gravity, and did not include a surfactant. Following a similar technique, we derive the equations for the two-dimensional spreading of a thin fluid with an insoluble surfactant on a horizontal surface. We begin with the Stokes equations in two dimensions: 


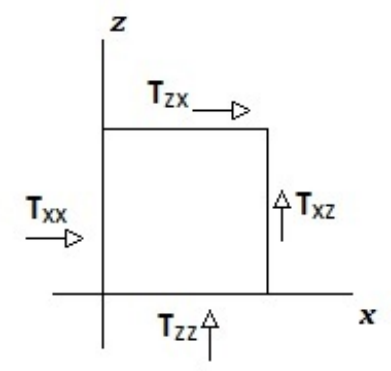

Figure 2: A square of fluid on the $x z$ plane with stress tensor components pictured.

$$
\begin{aligned}
-\partial_{x} p+\partial_{x} \tau_{x x}+\partial_{z} \tau_{x z} & =0 \\
-\partial_{z} p+\partial_{x} \tau_{z x}+\partial_{z} \tau_{z z} & =0 \\
u_{x}+w_{z} & =0
\end{aligned}
$$

where

$$
\tau_{i j}=2 A E^{\frac{1-\lambda}{\lambda}} \dot{\xi}_{i j}
$$

is the stress tensor component of the stress acting on the $i$ face of the fluid in the $j$ direction. For example, consider a cube of fluid on the $x z$ plane as shown in Figure 2. $\tau_{x z}$ would represent the stress acting on the $x$ side of the square tangentially in the $z$ direction. In (2), $A$ is a positive constant, $E=\left(\dot{\xi}_{i j} \dot{\xi}_{i j}\right)^{\frac{1}{2}}, \dot{\xi}_{i j}=\frac{1}{2}\left(\partial_{j} \vec{u}_{i}+\partial_{i} \vec{u}_{j}\right)$ (using tensor notation with repeated subscripts denoting summation), and $\vec{u}_{i}$ is the $i$ component of the velocity vector $\vec{u}=\langle u(x, z), w(x, z)\rangle$. Applying the lubrication approximation, we simplify $\dot{\xi}_{i j} \dot{\xi}_{i j}=\frac{1}{2}\left(\partial_{z} u\right)^{2}$.

Equation (2) is also dependent on $\lambda$, which is a parameter related to the powerlaw index of the fluid. If a fluid has a $\lambda$-index equal to 1 , then it signifies the fluid is Newtonian. A fluid with $\lambda>1$ indicates the fluid is shear-thinning, while shearthickening fluids have a value of $\lambda<1$. The power-law relationship is discussed in more detail in Section 3.3.

Additionally, using the lubrication approximation in (1a), we look at the order of magnitude of $\tau_{x x}$ and $\tau_{x z}$ :

$$
\begin{gathered}
\tau_{x x} \sim \dot{\xi}_{x x}=\frac{1}{2}\left(\partial_{x} u+\partial_{x} u\right)=\partial_{x} u \sim \frac{U}{L}, \\
\tau_{x z} \sim \dot{\xi}_{x z}=\frac{1}{2}\left(\partial_{z} u+\partial_{x} w\right) \sim \partial_{z} u \sim \frac{U}{H},
\end{gathered}
$$

where $U$ is the velocity scale of the fluid.

Since $H \ll L$, we know $\frac{U}{L} \ll \frac{U}{H}$ and consequently, $\tau_{x x} \ll \tau_{x z}$. So, (1a) is reduced to $-\partial_{x} p+\partial_{z} \tau_{x z}=0$. In a similar fashion, we look at the scalings of components of the stress tensor terms in $(1 \mathrm{~b})$ :

$$
\tau_{z x} \sim \dot{\xi}_{z x}=\frac{1}{2}\left(\partial_{x} w+\partial_{z} u\right) \sim \partial_{z} u \sim \frac{U}{H},
$$




$$
\tau_{z z} \sim \dot{\xi}_{z z}=\frac{1}{2}\left(\partial_{z} w+\partial_{z} w\right) \sim \partial_{z} w \sim \frac{W}{H} \sim \frac{\epsilon U}{H} \ll 1 .
$$

The magnitude of $\tau_{z x}$ is much less than the magnitude of the pressure term $\frac{L}{U}$ obtained from balancing leading order terms with the non-dimensionalization of (1a). Furthermore, we find that the $\tau_{z z}$ term is negligible. With these observations, we reduce (1) to

$$
\begin{aligned}
-\partial_{x} p+\partial_{z} \tau_{x z} & =0 \\
\partial_{z} p & =0 .
\end{aligned}
$$

Substituting in the stress tensor component (2) gives the reduced Stokes equations:

$$
\begin{aligned}
-p_{x}+\left(A u_{z}\left(\frac{1}{\sqrt{2}} u_{z}\right)^{\frac{1-\lambda}{\lambda}}\right)_{z} & =0 \\
p_{z} & =0 .
\end{aligned}
$$

Equation (4) agrees with the work of Perazzo and Gratton in [17], disregarding the third dimension and components of gravity.

\subsection{Boundary Conditions}

- No-Slip: We assume the horizontal substrate at $z=0$ to be at rest, resulting in the no-slip boundary condition

$$
u=0 \text { at } z=0 .
$$

- Normal Stress: Assuming the pressure at the surface of the fluid equals the atmospheric pressure gives the normal stress boundary condition

$$
p=p_{\text {atm }} \text { at } z=h(x, t) .
$$

Traditionally, the normal stress boundary condition also takes into account the surface tension and curvature of the fluid since the force on the film from the atmosphere must be balanced with the pressure from the film at the top of the fluid. This can be expressed as $p=p_{\text {atm }}-\gamma \kappa$, where $\gamma$ is the surface tension of the fluid and $\kappa$ is the curvature. However, including these terms does not allow for similarity solutions to be found.

- Tangential Stress: The change in surface tension from the surfactant results in a stress on the surface of the film acting tangentially to the film, suggesting the tangential stress boundary condition

$$
\frac{\partial}{\partial x} \sigma(\Gamma)=A u_{z}\left(\frac{1}{\sqrt{2}} u_{z}\right)^{\frac{1-\lambda}{\lambda}} \text { at } z=h(x, t)
$$

\subsection{Integration}

To derive the thin film equations, we integrate the reduced Stokes equations from (4) and apply boundary conditions (5), (6), and (7).

Integrating (4b) with respect to $z$ once, applying the normal stress boundary condition (6), and taking the partial derivative with respect to $x$ results in 


$$
p_{x}=0 .
$$

Substituting $p_{x}=0$ into (4a), applying the tangential stress boundary condition (7) to solve for the constant of integration, and dividing through by constant variables to isolate $u_{z}$ gives

$$
u_{z}(x, z, t)=\frac{1}{A^{\lambda} \sqrt{2}^{\lambda-1}}\left(\sigma_{x}\right)^{\lambda}
$$

Integrating with respect to $z$ again and applying the no-slip boundary condition (5) yields

$$
u(x, z, t)=\frac{1}{A^{\lambda} \sqrt{2}^{\lambda-1}}\left(\sigma_{x}\right)^{\lambda} z .
$$

\subsection{Depth Average Velocity}

With a thin film, taking a depth-average of the velocity over the thickness of the film gives a good approximation of the velocity at any spatial point. We denote the depth average velocity as $\bar{u}$,

$$
\bar{u}=\frac{1}{h} \int_{0}^{h} u d z,
$$

where $h$ is the height of the film.

Evaluating $\bar{u}$ with the velocity from (8) results in the depth average velocity:

$$
\bar{u}=\frac{1}{h} \frac{1}{A^{\lambda} \sqrt{2}^{\lambda-1}}\left(\sigma_{x}\right)^{\lambda} \frac{h^{2}}{2} .
$$

\subsection{Conservation of Mass of Fluid}

Since the fluid is assumed to be incompressible, the mass of the fluid must be conserved. Considering two spatial points $x=a$ and $x=b$, we create a relationship between the net flux of the fluid through these two points and the height of the fluid:

$$
\frac{d}{d t} \int_{a}^{b} h d x=F_{a}-F_{b}
$$

where $F_{a}$ and $F_{b}$ are the respective fluxes, and can be written as $F_{a}=\left.h \bar{u}\right|_{x=a}$ and $F_{b}=\left.h \bar{u}\right|_{x=b}$. Using the Fundamental Theorem of Calculus, the right side of (10) can then be expressed as an integral as well, which gives

$$
\frac{d}{d t} \int_{a}^{b} h d x=\int_{b}^{a} \frac{\partial}{\partial x}(h \bar{u}) d x .
$$

With some integral arithmetic, we arrive at

$$
\int_{a}^{b} \frac{\partial}{\partial t} h+\frac{\partial}{\partial x}(h \bar{u}) d x=0 .
$$

Since the integral equals 0 over any two arbitrary points $a$ and $b$, we know the integrand must equal 0 by the du-Bois Reymond lemma (or the fundamental lemma of calculus of variations), implying

$$
h_{t}+(h \bar{u})_{x}=0 .
$$


Substituting the depth average velocity from (9) gives the equation for the evolution of the height of the fluid:

$$
h_{t}+\left(\frac{1}{A^{\lambda} \sqrt{2}^{\lambda-1}}\left(\sigma_{x}\right)^{\lambda} \frac{h^{2}}{2}\right)_{x}=0 .
$$

\subsection{Conservation of Mass of Surfactant}

Similar to the mass of the fluid being conserved, the mass of the surfactant will be conserved as well. Using the insolubility of the surfactant, we write a relationship of

$$
\frac{d}{d t} \int_{a}^{b} \Gamma d x=F_{a}^{\Gamma}-F_{b}^{\Gamma}
$$

where $F_{a}^{\Gamma}$ and $F_{b}^{\Gamma}$ are the amounts of the surfactant passing through two spatial points $x=a$ and $x=b$ in the fluid. These can be written as $F_{a}^{\Gamma}=\left.\Gamma u\right|_{z=h(a, t)}$ and $F_{b}^{\Gamma}=$ $\left.\Gamma u\right|_{z=h(b, t)}$ respectively. We use the fluid velocity (8) evaluated at $z=h$ at both points instead of using the depth average velocity since the insoluble surfactant will be at the top of the fluid due to its immiscible nature. We write the right side of (12) as an integral using the Fundamental Theorem of Calculus,

$$
\frac{d}{d t} \int_{a}^{b} \Gamma d x=\int_{b}^{a} \frac{\partial}{\partial x}(\Gamma u) d x
$$

which can be rewritten as

$$
\int_{a}^{b} \frac{\partial}{\partial t} \Gamma+\frac{\partial}{\partial x}(\Gamma u) d x=0 .
$$

Again, since the integral evaluated at any two points $a$ and $b$ will be 0 , we know the integrand must be 0 by the du-Bois Reymond lemma. This leaves us with the equation for the spreading of the surfactant after we substitute in the velocity (8) at $z=h$ :

$$
\Gamma_{t}+\left(\frac{\Gamma}{A^{\lambda} \sqrt{2}^{\lambda-1}}\left(\sigma_{x}\right)^{\lambda} h\right)_{x}=0 .
$$

\subsection{Non-Dimensionalization}

Consider the change of variables: $x=L \hat{x}, z=H \hat{z}, t=T \hat{t}, \sigma=S \hat{\sigma}, \Gamma=C \hat{\Gamma}$, where $\hat{x}$ and $\hat{z}$ are non-dimensional spatial variables, $\hat{t}$ is the non-dimensional time variable, $\hat{\sigma}$ is the non-dimensional surface tension, $\hat{\Gamma}$ is the non-dimensional surfactant concentration, and $L, H, T, S, C$ are dimensional scalings of each respective variable.

Implementing these into (11) and (13) yields

$$
\begin{aligned}
& \frac{H}{T} \hat{h}_{\hat{t}}+\frac{H^{2}}{L}\left(\frac{S}{L}\right)^{\lambda} \frac{1}{A^{\lambda} \sqrt{2}^{\lambda-1}}\left(\left(\hat{\sigma}_{\hat{x}}\right)^{\lambda} \frac{\hat{h}^{2}}{2}\right)_{\hat{x}}=0, \\
& \frac{C}{T} \hat{\Gamma}_{\hat{t}}+\frac{H C}{L}\left(\frac{S}{L}\right)^{\lambda} \frac{1}{A^{\lambda} \sqrt{2}^{\lambda-1}}\left(\hat{\Gamma}\left(\hat{\sigma}_{\hat{x}}\right)^{\lambda} \hat{h}\right)_{\hat{x}}=0 .
\end{aligned}
$$

Balancing the coefficients of each equation leads to the same relation

$$
T=\frac{L^{\lambda+1} A^{\lambda} \sqrt{2}^{\lambda-1}}{S^{\lambda} H} .
$$


Multiplying (14a) and (14b) by $\frac{T}{H}$ and $\frac{T}{C}$ respectively and dropping the hats gives the non-dimensional equations

$$
\begin{aligned}
& h_{t}+\left(\left(\sigma_{x}\right)^{\lambda} \frac{h^{2}}{2}\right)_{x}=0, \\
& \Gamma_{t}+\left(\Gamma\left(\sigma_{x}\right)^{\lambda} h\right)_{x}=0 .
\end{aligned}
$$

The surface tension, $\sigma=\sigma(\Gamma)$, of the fluid is only dependent on the surfactant concentration, $\Gamma$. We use the linear equation of state:

$$
\sigma(\Gamma)=1-\Gamma
$$

which has been commonly used in mathematical studies, [5, 11, 14, 15]. Taking the derivative of $\sigma(\Gamma)$ with respect to $x$ results in $\sigma_{x}=-\Gamma_{x}$. Substituting this into (15a) and (15b) provides the system:

$$
\begin{aligned}
& h_{t}+\left(\left(-\Gamma_{x}\right)^{\lambda} \frac{h^{2}}{2}\right)_{x}=0, \\
& \Gamma_{t}+\left(\Gamma\left(-\Gamma_{x}\right)^{\lambda} h\right)_{x}=0 .
\end{aligned}
$$

We apply the initial conditions

$$
\begin{aligned}
& h(x, 0)=h_{0}(x)=1 \\
& \Gamma(x, 0)=\Gamma_{0}(x)= \begin{cases}1-x^{2}, & |x|<1 \\
0, & \text { otherwise }\end{cases}
\end{aligned}
$$

and boundary conditions from the symmetry of the spreading

$$
h_{x}(0, t)=0, \quad \Gamma_{x}(0, t)=0 .
$$

to determine the two remaining boundary conditions determined from the jump condition, as identified in [8] and further explored in [18].

\subsection{Jump Conditions}

In surfactant-driven fluid flow, the surfactant causes a gradient in the surface tension of the fluid, resulting in Marangoni flow away from the surfactant. Since the surfactant is a collection of discrete molecules, there is going to be a point in the surfactant profile, defined as the leading edge $x_{0}(t)$, where there is a transition from the region with surfactant to the region without surfactant. This suggests

$$
\Gamma\left(x_{0}, 0\right)=0
$$

as one of the boundary conditions since the surfactant concentration maintains compact support shown in [18]. With the shift in the surfactant profile from $\Gamma>0$ to $\Gamma=0$, there is a resulting jump in $\Gamma_{x}$ which triggers a jump in $h$. Employing the Rankine-Hugoniot jump condition and following an argument similar to those in $[7,19]$, we are able to determine jump conditions for our system: 


$$
\frac{d}{d t} \Gamma\left(x_{0}(t), t\right)=\dot{x}_{0} \Gamma_{x}+\Gamma_{t}=0 .
$$

which resembles the Linear Transport Equation, where $s=\dot{x}_{0}$ is the wave speed. Substituting (19) into (17b) yields

$$
\dot{x}_{0} \Gamma_{x}=\left(h \Gamma\left(-\Gamma_{x}\right)^{\lambda}\right)_{x} .
$$

Expanding the right hand side and noting $\Gamma\left(x_{0}(t), t\right)=0$ implies

$$
\dot{x}_{0} \Gamma_{x}=h \Gamma_{x}\left(-\Gamma_{x}\right)^{\lambda} .
$$

Dividing through by $\Gamma_{x} \neq 0$ results in

$$
\dot{x}_{0}=h\left(-\Gamma_{x}\right)^{\lambda}
$$

and suggests the transport speed is $s=h\left(-\Gamma_{x}\right)^{\lambda}$. We define

$$
\begin{array}{r}
h^{-}=\lim _{x \rightarrow x_{0}^{-}} h, \quad h^{+}=\lim _{x \rightarrow x_{0}^{+}} h, \\
\Gamma_{x}^{-}=\lim _{x \rightarrow x_{0}^{-}} \Gamma_{x}<0, \quad \Gamma_{x}^{+}=\lim _{x \rightarrow x_{0}^{+}} \Gamma_{x}=0 .
\end{array}
$$

The leading edge of the surfactant moves with the surface velocity of the fluid. Therefore, the transport speed is $s=h^{-}\left(-\Gamma_{x}^{-}\right)^{\lambda}$. From there, we know

$$
s[h]=\left[\frac{1}{2} h^{2}\left(-\Gamma_{x}\right)^{\lambda}\right],
$$

where [ ] denotes a jump. For example, $[h]=\left(h^{+}-h^{-}\right)$.

Substituting in $s=h\left(-\Gamma_{x}\right)^{\lambda}$ and expanding the $h$ and $\Gamma_{x}$ terms, we are left with

$$
h^{-}\left(-\Gamma_{x}^{-}\right)^{\lambda}\left(h^{+}-h^{-}\right)=\frac{1}{2}\left(\left(h^{+}\right)^{2}\left(-\Gamma_{x}^{+}\right)^{\lambda}-\left(h^{-}\right)^{2}\left(-\Gamma_{x}^{-}\right)^{\lambda}\right) .
$$

Applying (20b) and dividing through by $h^{-}\left(-\Gamma_{x}^{-}\right)^{\lambda} \neq 0$ leaves

$$
\left(h^{+}-h^{-}\right)=-\frac{1}{2} h^{-},
$$

which reduces down to a jump condition at $x=x_{0}(t)$ :

$$
h^{-}=2 h^{+} .
$$

Thus, the height profile will jump to twice its initial height due to the jump in $\Gamma_{x}$ and the final boundary condition is $h\left(x_{0}, t\right)=2$. The jump conditions found for Newtonian fluids with surfactant in $[7,8,19]$ are in agreement with the jump conditions for a non-Newtonian fluid, (21).

Therefore, the boundary conditions for (17) are

$$
\begin{array}{cc}
h\left(x_{0}, t\right)=2, & \Gamma\left(x_{0}, t\right)=0, \\
h_{x}(0, t)=0, & \Gamma_{x}(0, t)=0 .
\end{array}
$$




\section{Numerical Method}

In this section, we discuss the numerical method used to investigate the behavior of the height and surfactant profiles.

\subsection{Numerical Method}

Since the leading edge $x_{0}$ is not set, we will solve a moving boundary problem as the domain where the solution is defined, $\left[0<x<x_{0}\right.$ ], evolves with time. We define a new spatial variable, $\xi=\frac{x}{x_{0}(t)}$, which uses the leading edge of the surfactant profile to scale the domain for the height and surfactant profiles (17) to the domain of [0,1]. A similar argument has been employed by Peterson and Shearer, [18]. Consider

$$
\widetilde{h}(\xi, t)=h(x, t), \quad \widetilde{\Gamma}(\xi, t)=\Gamma(x, t), \quad \xi=\frac{x}{x_{0}(t)} .
$$

Dropping the , (17a) and (17b) become

$$
\begin{aligned}
& h_{t}-\frac{\dot{x}_{0}}{x_{0}} \xi h_{\xi}=\frac{1}{x_{0}^{\lambda+1}}\left(\frac{h^{2}}{2}\left(-\Gamma_{\xi}\right)^{\lambda}\right)_{\xi} \\
& \Gamma_{t}-\frac{\dot{x}_{0}}{x_{0}} \xi \Gamma_{\xi}=\frac{1}{x_{0}^{\lambda+1}}\left(h \Gamma\left(-\Gamma_{\xi}\right)^{\lambda}\right)_{\xi}
\end{aligned}
$$

on $0 \leq \xi \leq 1$ where the corresponding initial conditions are

$$
\begin{aligned}
& h(\xi, 0)=1, \\
& \Gamma(\xi, 0)=\left\{\begin{array}{ll}
1-\xi^{2} & \xi<1 \\
0 & \xi \geq 1
\end{array},\right.
\end{aligned}
$$

and boundary conditions are

$$
\begin{gathered}
h_{\xi}(0, t)=0, \quad \Gamma_{\xi}(0, t)=0, \\
h(1, t)=2, \quad \Gamma(1, t)=0 .
\end{gathered}
$$

We solve for the speed of the leading edge of the surfactant, $\dot{x}_{0}$, by evaluating $(23 \mathrm{~b})$ at $\xi=1$ and noting $\Gamma(1, t)=0$ and $\Gamma_{t}(1, t)=0$ to find

$$
-\left.\frac{\dot{x}_{0}}{x_{0}} \Gamma_{\xi}\right|_{\xi=1^{-}}=\left.\frac{1}{x_{0}^{\lambda+1}}\left(h \Gamma_{\xi}\left(-\Gamma_{\xi}\right)^{\lambda}\right)\right|_{\xi=1^{-}} .
$$

Multiplying through by $x_{0}$ and dividing both sides through by $\Gamma_{\xi} \neq 0$ yields

$$
\dot{x}_{0}=-\left.\frac{1}{x_{0}^{\lambda}} h\left(-\Gamma_{\xi}\right)^{\lambda}\right|_{\xi=1^{-}} .
$$

\subsection{Numerical Scheme}

We use a finite difference method to numerically simulate the transformed system (23) and (26).

Let $h_{j}^{n} \approx h(j \Delta \xi, n \Delta t)$ and $\Gamma_{j}^{n} \approx \Gamma(j \Delta \xi, n \Delta t)$ where $\Delta \xi=\frac{1}{N}$ and $N$ is the number of grid points (we used $N=1000$ for our simulations). We use a centered difference 
in space and backward difference in time to discretize the system. Since we used a centered difference in space, we use LU decomposition to find the 3 -banded $n \times n$ diagonal Jacobian matrix. The discretized system of (23) and (26) are implicitly defined as the system

$$
\begin{aligned}
& h_{j}^{n+1}-h_{j}^{n}-\Delta t \xi_{j} \frac{x_{0}^{n+1}-x_{0}^{n}}{x_{0}^{n} \Delta t}\left(\frac{h_{j+1}^{n+1}-h_{j}^{n+1}}{\Delta \xi}\right)+ \\
& \frac{\Delta t}{\left(x_{0}^{n}\right)^{\lambda+1} \Delta \xi}\left[\frac{1}{2}\left(\frac{h_{j+1}^{n+1}+h_{j}^{n+1}}{2}\right)\left(\frac{\Gamma_{j}^{n+1}-\Gamma_{j+1}^{n+1}}{\Delta \xi}\right)^{\lambda}-\frac{1}{2}\left(\frac{h_{j-1}^{n+1}+h_{j}^{n+1}}{2}\right)\left(\frac{\Gamma_{j-1}^{n+1}-\Gamma_{j}^{n+1}}{\Delta \xi}\right)^{\lambda}\right]=0,
\end{aligned}
$$

$$
\begin{aligned}
\Gamma_{j}^{n+1}-\Gamma_{j}^{n}-\Delta t \xi_{j} \frac{x_{0}^{n+1}-x_{0}^{n}}{x_{0}^{n} \Delta t}\left(\frac{\Gamma_{j+1}^{n+1}-\Gamma_{j}^{n+1}}{\Delta \xi}\right)+ \\
\frac{\Delta t}{\left(x_{0}^{n}\right)^{\lambda+1} \Delta \xi}\left[\left(\frac{h_{j+1}^{n+1}+h_{j}^{n+1}}{2}\right)\left(\frac{\Gamma_{j+1}^{n+1}+\Gamma_{j}^{n+1}}{2}\right)\left(\frac{\Gamma_{j}^{n+1}-\Gamma_{j+1}^{n+1}}{\Delta \xi}\right)^{\lambda}\right]- \\
\frac{\Delta t}{\left(x_{0}^{n}\right)^{\lambda+1} \Delta \xi}\left[\left(\frac{h_{j-1}^{n+1}+h_{j}^{n+1}}{2}\right)\left(\frac{\Gamma_{j-1}^{n+1}+\Gamma_{j}^{n+1}}{2}\right)\left(\frac{\Gamma_{j-1}^{n+1}-\Gamma_{j}^{n+1}}{\Delta \xi}\right)^{\lambda}\right]=0
\end{aligned}
$$

$x_{0}^{n+1}=x_{0}^{n}+h_{j}^{n+1} \frac{\Delta t}{\left(x_{0}^{n}\right)^{\lambda}}\left(\frac{\Gamma_{j-1}^{n+1}}{\Delta \xi}\right)^{\lambda}$.

Initial and boundary conditions corresponding to (24) and (25) include

$$
\begin{array}{cc}
h_{j}^{0}=1, & \Gamma_{j}^{0}=1-\xi_{j}^{2}, \\
h_{0}^{n}=h_{1}^{n}, & \Gamma_{0}^{n}=\Gamma_{1}^{n}, \\
h_{N}^{n}=2, & \Gamma_{N}^{n}=0 .
\end{array}
$$

Finally, we employ Newton's method to solve for solutions of (27).

\subsection{Varying $\lambda$}

We consider a power-law relation due to the relative simplicity of the apparent viscosity $\eta$, which is commonly expressed as [1]

$$
\eta=K|\dot{\gamma}|^{n-1}
$$

where $K$ is a positive constant, $\dot{\gamma}$ is the shear rate, and $n$ is the deviation from the Newtonian fluid power-law index of $n=1$. For example, a fluid with a power-law index of $n=1$ would be a Newtonian fluid, $n>1$ would indicate a shear-thickening fluid, and $n<1$ would signify a shear-thinning fluid. Table 1 gives values for the power-law indices for several non-Newtonian fluids with $n<1$.

In Section 2.1, we defined the stress tensor (2). The stress tensor can be related to the apparent viscosity with 
Table 1: Well-known shear-thinning fluids, [1].

\begin{tabular}{|c|c|c|}
\hline \hline Material & $\mathrm{K}$ & $n$ \\
\hline Ball-point pen ink & 10 & 0.85 \\
Fabric Conditioner & 10 & 0.6 \\
Molten Chocolate & 50 & 0.5 \\
Toothpaste & 35 & 0.3 \\
Skin cream & 45 & 0.1 \\
\hline
\end{tabular}

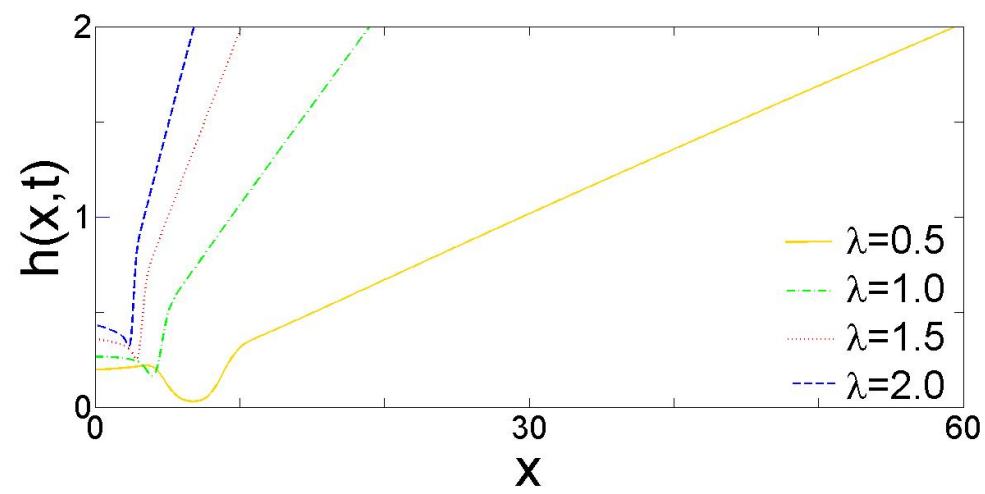

Figure 3: The height profile $h(x, t)$ for $\lambda=0.5,1.0,1.5$, and 2.0 for $t=1000$ with $\Delta x=.001$.

$$
\tau=\eta \dot{\xi}_{i j}, \quad \text { where } \eta=2 A E^{\frac{1-\lambda}{\lambda}} .
$$

Letting $K=2 A, \dot{\gamma}=E$, and $n=\frac{1}{\lambda}$, we make substitutions into (29) and recover (28).

Figure 3 illustrates the influence of $\lambda$ on the height and surfactant profiles. This profile is in agreement with the idea that fluids with a higher $\lambda$-index flow more slowly than fluids with a lower $\lambda$-index, corresponding to shear-thinning fluids $(n<1)$ flowing slower than shear-thickening fluids $(n>1)$.

\section{Similarity Solutions}

In this section, we explore the solutions near the leading edge of surfactant in the height and surfactant profiles with a similarity scaling analysis and find the explicit similarity solutions. We also compare our numerics and similarity solutions to previous work with Newtonian fluids, $[9,18]$.

\subsection{Similarity Scaling}

Consider the spatial variables to be scaled by time:

$$
h=t^{\mu} H(\rho), \quad \Gamma=t^{\nu} G(\rho), \quad \text { where } \rho=\frac{x}{t^{\beta}} .
$$


Then, (17a) and (17b) become

$$
\begin{aligned}
& \mu t^{\mu-1} H-\beta t^{\mu-1} \rho H^{\prime}+t^{2 \mu+\nu \lambda-\beta \lambda-\beta}\left(\frac{H^{2}}{2}\left(-G^{\prime}\right)^{\lambda}\right)^{\prime}=0, \\
& \nu t^{\nu-1} G-\beta t^{\nu-1} \rho G^{\prime}+t^{\nu+\mu+\nu \lambda-\beta \lambda-\beta}\left(G H\left(-G^{\prime}\right)^{\lambda}\right)^{\prime}-0,
\end{aligned}
$$

where ' denotes $\frac{d}{d \rho}$. From balancing powers of $t$, we get the same relation from (31a) and $(31 \mathrm{~b})$ of

$$
0=\mu+1+\nu \lambda-\beta(\lambda+1)
$$

The conservation of the surfactant,

$$
\int_{0}^{t^{\beta}} \Gamma d x=\int_{0}^{1} t^{\nu+\beta} G(\rho) d \rho
$$

suggests

$$
\nu+\beta=0
$$

Also, the boundary condition of $h(1, t)=2$ from $(25 \mathrm{~b})$ implies

$$
\mu=0 \text {. }
$$

From (32), (33), and (34), we find

$$
\mu=0, \quad \nu=-\frac{1}{2 \lambda+1}, \quad \beta=\frac{1}{2 \lambda+1} .
$$

Implementing these scalings into (31a) and (31b) results in

$$
\begin{gathered}
-\frac{1}{2 \lambda+1} \rho H^{\prime}+\left(\frac{H^{2}}{2}\left(-G^{\prime}\right)^{\lambda}\right)^{\prime}=0, \\
-\frac{1}{2 \lambda+1}(\rho G)^{\prime}+\left(H G\left(-G^{\prime}\right)^{\lambda}\right)^{\prime}=0 .
\end{gathered}
$$

Integrating (36b) yields

$$
-\frac{1}{2 \lambda+1}(\rho G)+H G\left(-G^{\prime}\right)^{\lambda}=k
$$

where $k$ is a constant; however, since we know $k$ is constant for all values of $H$ and $G$ on the domain $\left[0, x_{0}\right]$ and also that $G(\rho)=0$ at $x=x_{0}$ from $(22 \mathrm{a})$, we determine $k=0$.

From here, we pull out $G$ to get:

$$
G\left(-\frac{1}{2 \lambda+1} \rho+H\left(-G^{\prime}\right)^{\lambda}\right)=0
$$

Since $G \not \equiv 0$, we know

$$
H\left(-G^{\prime}\right)^{\lambda}=\frac{1}{2 \lambda+1} \rho
$$

Substituting into (36a) results in

$$
-\frac{1}{2 \lambda+1} \rho H^{\prime}+\left(\frac{H \rho}{2(2 \lambda+1)}\right)^{\prime}=0,
$$




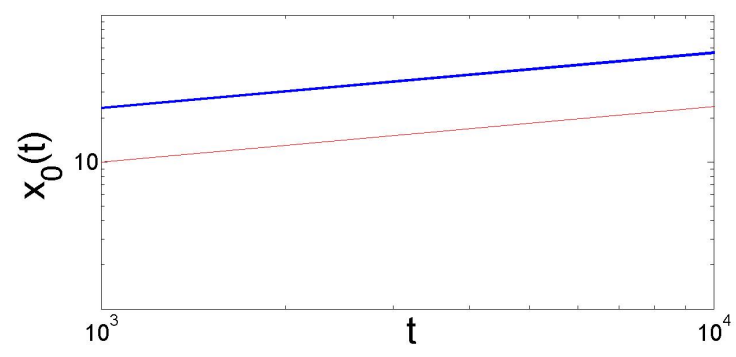

Figure 4: Log-log plot of the leading edge of surfactant $x_{0}(t)$ vs. $t$ with data from $\lambda=1$. The comparison line (red) has slope $1 / 3$.

which simplifies to

$$
H=\rho H^{\prime}
$$

Using separation of variables and applying the jump condition indicates

$$
H(\rho)=2 \rho .
$$

Substituting (38) into (36b) gives

$$
G^{\prime}=-\left(\frac{1}{2(2 \lambda+1)}\right)^{\frac{1}{\lambda}} .
$$

Integrating and applying the initial condition of $G(1)=0$, we find

$$
G(\rho)=\left(\frac{1}{2(2 \lambda+1)}\right)^{\frac{1}{\lambda}}(1-\rho) .
$$

Letting $\lambda=1$ transforms both (38) and (39) into the same equations found for the Newtonian case in [10].

When we compare the spatial variable $\rho=\frac{x}{t^{\beta}}$ to the spatial variable $\xi=\frac{x}{x_{0}(t)}$ in Section 3.1, we are examining the independence of $x_{0}(t)$ on $t$ as illustrated in the log-log plot in Figure 4 with data from the $\lambda=1$ case (where $\beta=\frac{1}{3}$ from equation (35)). The resulting graph is a straight line (in blue) with slope $\frac{1}{3}$. For comparison, a red line with slope $\frac{1}{3}$ is also plotted. Thus, the leading edge of the surfactant profile is similar to the scaled time, $x_{0}(t) \sim t^{1 / 3}$. Analogous arguments can easily be made for $\lambda=.5$ and $\lambda=1.5$ to show $x_{0}(t) \sim t^{1 / 2}$ and $x_{0}(t) \sim t^{1 / 4}$ for their respective cases. Accordingly, the numerical simulations verify the scalings of the spatial variable with respect to time as found above.

\subsection{Similarity Solutions}

Consider (30) to be scaled with constants

$$
h=a t^{\mu} H(\rho), \quad \Gamma=b t^{\nu} G(\rho), \quad \text { where } \rho=\frac{x}{D t^{\beta}},
$$

which lets us bring in the initial mass of the fluid and incorporate the initial surfactant mass into the similarity solutions. Substituting in the exponents found in (35), (40) becomes

$$
h(x, t)=a H(\rho), \quad \Gamma(x, t)=b t^{-\frac{1}{2 \lambda+1}} G(\rho), \quad \rho=\frac{x}{(D t)^{\frac{1}{2 \lambda+1}}} .
$$


Substituting (41) into (17a) and (17b) yields

$$
\begin{array}{r}
-\frac{1}{2 \lambda+1} a \rho H^{\prime}-\frac{a^{2}}{D^{\frac{1}{3}}}\left(\frac{b}{D^{\frac{1}{3}}}\right)^{\lambda}\left(\frac{H^{2}}{2}\left(-G^{\prime}\right)^{\lambda}\right)^{\prime}=0, \\
-\frac{1}{2 \lambda+1} b G-\frac{1}{2 \lambda+1} b \rho H^{\prime}-\frac{a b}{D^{\frac{1}{3}}}\left(\frac{b}{D^{\frac{1}{3}}}\right)^{\lambda}\left(H G\left(-G^{\prime}\right)^{\lambda}\right)^{\prime}=0 .
\end{array}
$$

We relate our scaling coefficients in both equations to find the same relationship,

$$
b^{\lambda}=\frac{D^{\frac{\lambda+1}{3}}}{a} .
$$

The initial condition (18a) implies $a=1$. Thus, $b^{\lambda}=D^{\frac{\lambda+1}{3}}$, where $D$ is determined by a relation of the conservation of the mass of the surfactant

$$
\int_{0}^{1} \Gamma_{0} d \xi=\int_{0}^{x_{0}(t)} \Gamma d x
$$

With the initial condition (18b), we find

$$
D=\left(2(2 \lambda+1)\left(\frac{4}{3}\right)^{\lambda}\right)^{\frac{3 \lambda(2 \lambda+1)}{2 \lambda^{2}+6 \lambda+1}}
$$

Our explicit solutions are

$$
\begin{aligned}
& h(\rho, t)=2 \rho \\
& \Gamma(\rho, t)=\left[\left(2(2 \lambda+1)\left(\frac{4}{3}\right)^{\lambda}\right)^{\frac{3 \lambda(2 \lambda+1)}{2 \lambda^{2}+6 \lambda+1}}\right]^{\frac{\lambda+1}{3 \lambda}}\left(\frac{1}{2(2 \lambda+1)}\right)^{\frac{1}{\lambda}}(1-\rho) t^{-\frac{1}{2 \lambda+1}} .
\end{aligned}
$$

The explicit similarity solutions are plotted in Figure 5, Figure 6, and Figure 7 for $\lambda=0.5, \lambda=1.0$, and $\lambda=1.5$ respectively. Setting $\lambda=1$ again returns the similarity solutions back to the Newtonian case, which are the same explicit solutions found in [10].

Another interesting note is that the similarity solution for the height profile will be the same for any value of $\lambda$, whereas the similarity solution for the surfactant profile is dependent on $\lambda$ and accordingly varies for fluids with differing values of $\lambda$.

\section{Discussion}

We conduct a numerical analysis for our system of nonlinear equations for thin film with surfactant, (17), which ignores the physical effects of gravity, capillarity, and surface diffusion. Although incorporating these physical elements would provide a more comprehensive picture, they prevent us from finding similarity solutions. Additionally, we could have employed axi-symmetric coordinates for the entirety of this paper; however, an analogous argument can be made for this coordinate system.

While we were able to determine similarity scalings for the outer region of our solutions, finding a general case for the similarity scalings at the inner region of the height and surfactant profiles have proved to be more difficult due to their dependency on $\lambda$. 

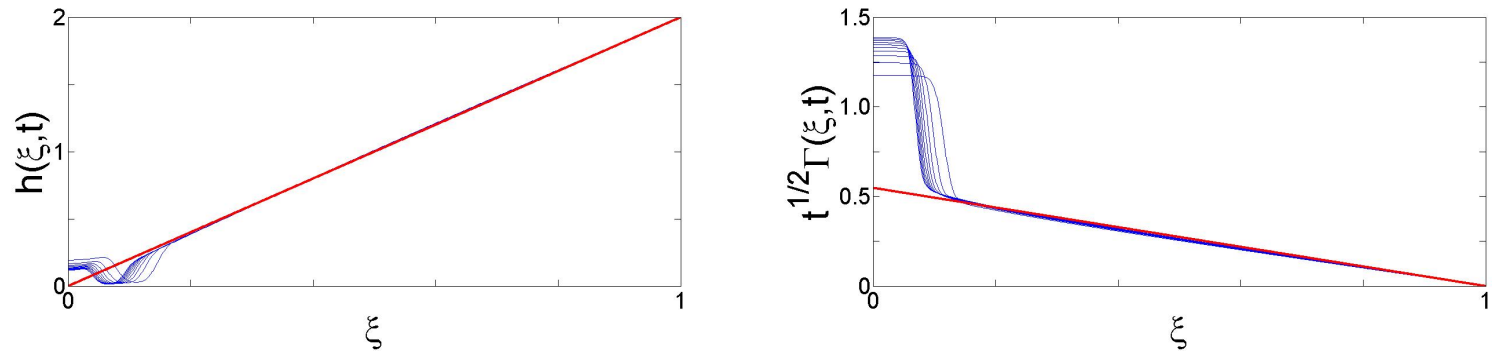

Figure 5: The height profile $h(\xi, t)$ and scaled surfactant profile $t^{1 / 2} \Gamma(\xi, t)$ plotted with their similarity solutions in red for $\lambda=0.5$ on $1000<t<10000$ with $\Delta \xi=.001$.
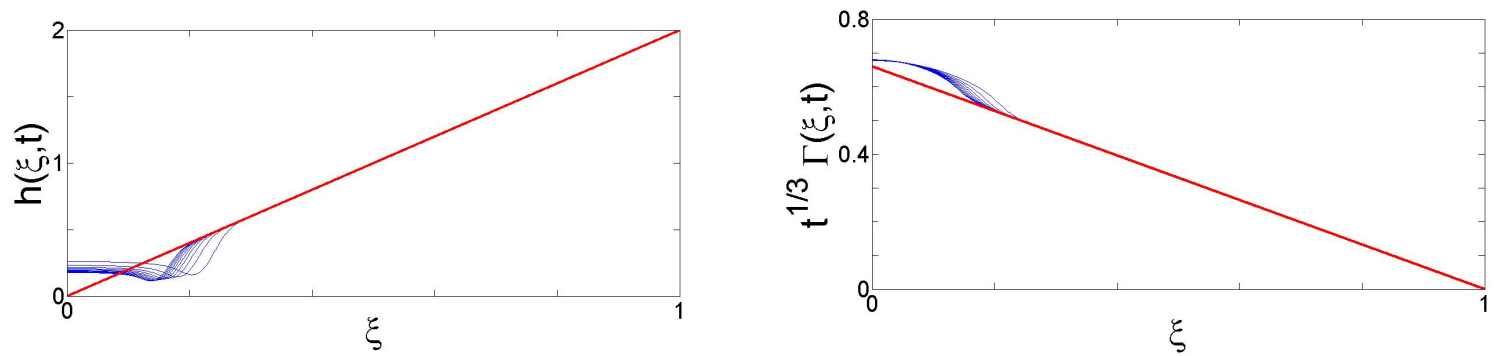

Figure 6: The height profile $h(\xi, t)$ and scaled surfactant profile $t^{1 / 3} \Gamma(\xi, t)$ plotted with their similarity solutions in red for $\lambda=1.0$ on $1000<t<10000$ with $\Delta \xi=.001$.
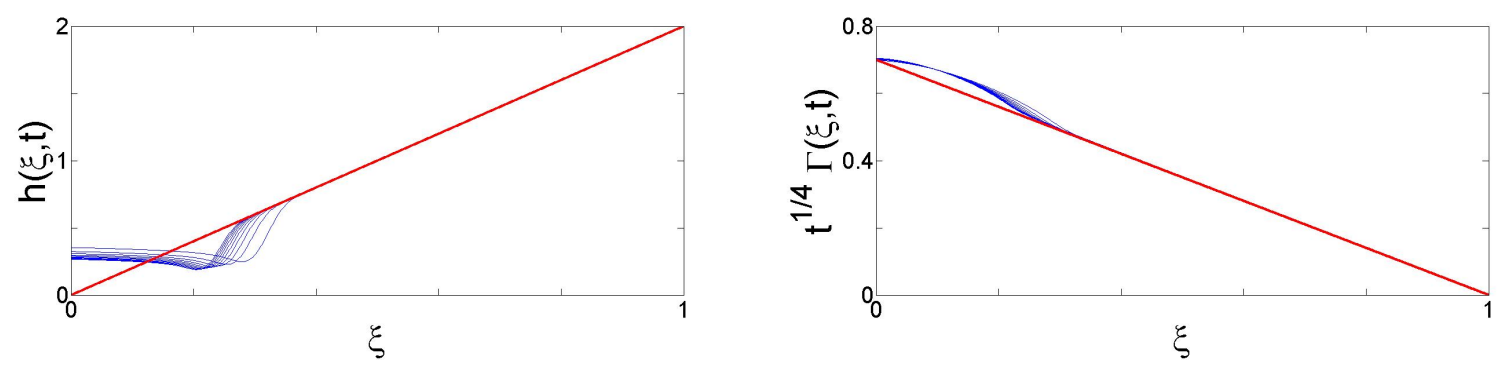

Figure 7: The height profile $h(\xi, t)$ and scaled surfactant profile $t^{1 / 4} \Gamma(\xi, t)$ plotted with their similarity solutions in red for $\lambda=1.5$ on $1000<t<10000$ with $\Delta \xi=.001$. 


\section{Acknowledgements}

This work was supported by the Centre College Faculty Development Committee. The author would like to thank Dr. Ellen Swanson for all of her advice and guidance throughout this process in addition to all the cookies she kept in her office. Also, thanks to Chang He for her helpful discussions and suggestions.

\section{References}

[1] H.A. BARNES, J.F. HUTTON, K. WALTERS. An Introduction to Rheology. Elsevier Science Publishers B.V., Amsterdam (1989).

[2] J. BULL, L. NELSON, J. WALSH, M. GLUCKBERGER, S. SCHÜRCH, J. GROTBERG. Surfactant-spreading and surface-compression disturbance on a thin viscous film. J. Biomech. Eng., 121:89-98 (1999).

[3] R.P. CHHABRA, J.F. RICHARDSON. Non-Newtonian Flow in the Process Industries: Fundamentals and Engineering Applications. Butterworth-Heinemann, Oxford (1999).

[4] R. CRASTER, O. MATAR. Numerical simulations of fingering instabilities in surfactant-driven thin films. Phys. Fluids 18, 032103 (2006).

[5] B.D. EDMONSTONE, O.K. MATAR, R.V. CRASTER. Flow of surfactant-laden thin films down an inclined plane Journal of Engineering Mathematics, 50:141-156 (2004).

[6] D.W. FALlEST, A.M. LICHTENBERGER, C. FOX, K.E. DANIELS. Fluorescent visualization of a spreading surfactant. New J. Phys. 12, 073029 (2010).

[7] D.P. GAVER, J.B. GROTBERG. Droplet spreading on a thin viscous film. J. Fluid Mech., 235:399-414 (1992).

[8] D.P. GAVER, J.B. GROTBERG. The dynamics of a localized surfactant on a thin film. J. Fluid Mech., 213:127-148 (1990).

[9] O.E. JENSEN. Self-similar, surfactant-driven flows. Phys. Fluids, 6:1084-1094 (1994).

[10] O.E. JENSEN, J.B. GROTBERG. Insoluble surfactant spreading on a thin viscous film: shock evolution and film rupture. J. Fluid Mech., 240:259-288 (1992).

[11] O.E. JENSEN, J.B. GROTBERG. The spreading of heat or soluble surfactant along a thin liquid film. Phys. Fluids A, 5 (1993).

[12] O.E. JENSEN, S. NAIRE. The spreading and stability of surfactant-laden drop on a prewetted substrate. J. Fluid Mech., 554:5-24 (2006).

[13] W.A. KHAN, M.J. UDDIN, A.I. ISMAIL. Free convection of non-Newtonian nanofluids in porous media with gyrotactic microorganisms. Transport in Porous Media, 97(2):241-252 (2013).

[14] R. LEVY, M. SHEARER, T. WITELSKI. Gravity-driven thin liquid films with insoluble surfactant: smooth traveling waves. Euro. J. Appl. Math, 18:679-708 (2007).

[15] O.K. MATAR, S.M. TROIAN. Spreading of a surfactant monolayer on a thin liquid film: Onset and evolution of digitated structures. Chaos, 9 (1999). 
[16] T.G. MYERS. Application of non-Newtonian models to thin film flow. Phys. Rev. E 72, 066302 (2005).

[17] C. PERAZZO and J. GRATTON. Thin film of non-Newtonian fluid on an incline Phys. Rev. E 67, 016307 (2003).

[18] E. PETERSON, M. SHEARER. Radial Spreading of a Surfactant on a Thin Liquid Film. Applied Mathematics Research eXpress, 2011:1-22 (2011).

[19] E. PETERSON, M. SHEARER. Simulation of spreading surfactant on a thin liquid film. Applied Mathematics and Computation, 218: 5157-5167 (2012).

[20] A. SADOUGHI, A. HIRSA, J. LOPEZ. Newtonian to non-Newtonian flow transition in lung surfactants. Amer. Phys. Society, 63rd Annual Meeting of the APS Division of Fluid Dynamics, (2010).

[21] G.K. SEevaratnam, Y. SOU, E. RAMÉ, L. M. WALKER, S. GAROFF. Dynamic wetting of shear thinning fluids. Phys. Fluids 19, 0121013 (2007).

[22] T.P. STEVENS, R.A. SINKIN. Surfactant Replacement Therapy. Chest, 131(5):1577-1582 (2007). 